\title{
CIS
}

Centro de Investigaciones Sociológicas

BAROMETRO ESPECIAL DE ABRIL 2020

\section{AVANCE DE RESULTADOS}

Estudio $n^{\circ} 3279$

Abril 2020 


\section{CIS}

Centro de Investigaciones Sociológicas

BARÓMETRO ESPECIAL DE ABRIL 2020

AVANCE DE RESULTADOS

FICHA TÉCNICA

Estudio $n^{\circ} 3279$

Abril 2020 


\section{ESTUDIO CIS N ${ }^{\circ} 3279$}

\section{BARÓMETRO ESPECIAL DE ABRIL 2020}

\section{FICHA TÉCNICA}

\section{Ámbito:}

Nacional.

\section{Universo:}

Población española de ambos sexos de 18 años y más.

\section{$\underline{\text { Tamaño de la muestra: }}$}

Diseñada: $\quad 3.000$ entrevistas.

Realizada: $\quad 3.000$ entrevistas.

\section{Afijación:}

Proporcional.

\section{Ponderación:}

No procede.

\section{Puntos de Muestreo:}

1.081 municipios y 50 provincias.

\section{Procedimiento de muestreo:}

Se ha procedido a la selección aleatoria de teléfonos fijos y móviles con un porcentaje del $58,1 \%$ y del $41,9 \%$, respectivamente. La selección de los individuos se ha llevado a cabo mediante la aplicación de cuotas de sexo y edad.

Los estratos se han formado por el cruce de las 17 comunidades autónomas y las dos ciudades autónomas, con el tamaño de hábitat, dividido en 7 categorías: menor o igual a 2.000 habitantes; de 2.001 a 10.000; de 10.001 a 50.000; de 50.001 a 100.000; de 100.001 a 400.000; de 400.001 a 1.000 .000 , y más de 1.000 .000 de habitantes.

Los cuestionarios se han aplicado mediante entrevista telefónica asistida por ordenador (CATI). 


\section{Error muestral:}

Para un nivel de confianza del $95,5 \%$ (dos sigmas) y $\mathrm{P}=\mathrm{Q}$, el error real es de $\pm 1,8 \%$ para el conjunto de la muestra y en el supuesto de muestreo aleatorio simple.

\section{Fecha de realización:}

La recogida de datos se ha realizado del 30 marzo al 7 de abril de 2020.

Dado que el CIS no dispone en estos momentos de un sistema para la realización de encuestas telefónicas, se contrató su realización mediante procedimiento de emergencia, después de pedir cuatro presupuestos a empresas especializadas. La empresa seleccionada fue INTERCAMPO S.A. Empresa formada por un equipo de profesionales con una amplia trayectoria en la investigación de mercados y opinión pública. Miembro de Insights + Analytics España, ESOMAR con certificaciones UNE-ISO 20.252, UNE-ISO 9.001, CCI-ESOMAR Internacional.

Para esta encuesta telefónica se ha aplicado el sistema CATI con grabación y gestión automática de llamadas, escucha y monitorización del trabajo. En esta ocasión, todos los entrevistadores han teletrabajado desde sus casas. 


\section{CIS}

Centro de Investigaciones Sociológicas

BARÓMETRO ESPECIAL DE ABRIL 2020

AVANCE DE RESULTADOS

DISTRIBUCIONES MARGINALES

Estudio $n^{\circ} 3279$

Abril 2020 


\section{NOTAS:}

A. Los/as entrevistadores/as no ofrecen las opciones de respuesta "NO LEER", pero las registran cuando son mencionadas espontáneamente por las personas entrevistadas.

B. Las categorías de respuesta que muestran porcentaje 0,0 han recibido al menos una mención en la encuesta pero, debido a la presentación de resultados con 1 solo decimal y al redondeo, no llega a visualizarse el valor real. Si las categorías no han sido respondidas por ninguna persona entrevistada, en los resultados se indica con un guión '-'.

C. En las preguntas P27R, P27aR, P28R y P33R se han incorporado los partidos con representación parlamentaria, autonómica, nacional y/o europea, y aquellos cuyas menciones representan el $0,5 \%$ o más de las respuestas espontáneas de las personas entrevistadas (aquellos partidos cuyas menciones no alcanzan el $0,5 \%$ han sido incluidos en la categoría 'Otro partido').

D. En la pregunta P27R la opción "Más País" incluye las respuestas a Más País y EQUO en las siguientes circunscripciones: Cádiz, Granada, Málaga, Sevilla, Asturias, Las Palmas, Santa Cruz de Tenerife, Barcelona, A Coruña, Pontevedra, Madrid, Murcia, Bizkaia; en Zaragoza "Más País-Chunta Aragonesista" recoge las menciones a la Chunta Aragonesista, Más País y EQUO; en las Illes Balears la opción "MÉS (PSM-Entesa)" recoge las menciones a MÉS y ERC; en Canarias la opción "CCa-NC" recoge las menciones a Coalición Canaria y Nueva Canarias; en la Comunitat Valenciana la opción "Més Compromís" incluye las menciones a Compromís, Más País y EQUO; la opción "NA+" incluye las menciones a PP, Ciudadanos y UPN, recogidas en la Comunidad Foral de Navarra; en el Principado de Asturias las menciones a Foro Asturias se incluyen dentro del "PP"; en el resto de circunscripciones la opción "Unidas Podemos" recoge las menciones a Podemos, Izquierda Unida (Esquerda Unida, ICV (Iniciativa per Catalunya-Verds)), Unidas Podemos, EQUO, En Común-Unidas Podemos y En Comú Podem. La variable VOTO+SIMPATÍA (C2a) sigue el mismo criterio.

E. En la pregunta P27aR y P32aR la opción "Más País" incluye las respuestas a Más País y EQUO en las siguientes circunscripciones: Cádiz, Granada, Málaga, Sevilla, Asturias, Las Palmas, Santa Cruz de Tenerife, Barcelona, A Coruña, Pontevedra, Madrid, Murcia, Bizkaia; en Zaragoza "Más País-Chunta Aragonesista" recoge las menciones a la Chunta Aragonesista, Más País y EQUO; en las Illes Balears la opción "MÉS (PSM-Entesa)" recoge las menciones a MÉS y ERC; en Canarias la opción "CCa-NC" recoge las menciones a Coalición Canaria y Nueva Canarias; en Cataluña la opción "En Comú Podem" incluye las menciones a En Comú Podem, Podemos, ICV (Iniciativa per Catalunya-Verds) y Unidas Podemos; en la Comunitat Valenciana la opción "Més Compromís" incluye las menciones a Compromís, Más País y EQUO; en Galicia "En Común-Unidas Podemos" agrupa las menciones a Podemos y Esquerda Unida; la opción "NA+" incluye las menciones a PP, Ciudadanos y UPN, recogidas en la Comunidad Foral de Navarra; en el Principado de Asturias las menciones a Foro Asturias se incluyen dentro del "PP"; en el resto de circunscripciones la opción "Unidas Podemos" recoge las menciones a Podemos, Izquierda Unida, Unidas Podemos y EQUO. 


\section{Pregunta OA}

\section{SEXO DE LA PERSONA ENTREVISTADA.}

\begin{tabular}{lr}
\hline Hombre & 48,5 \\
\hline Mujer & 51,5 \\
\hline (N) & $(3.000)$ \\
\hline
\end{tabular}

\section{Pregunta OB}

¿Qué edad tiene Ud.?

\begin{tabular}{lr}
\hline De 18 a 24 años & 7,9 \\
\hline De 25 a 34 años & 12,7 \\
\hline De 35 a 44 años & 18,8 \\
\hline De 45 a 54 años & 19,6 \\
\hline De 55 a 64 años & 16,4 \\
\hline 65 y más años & 24,7 \\
\hline (N) & $(3.000)$ \\
\hline
\end{tabular}

\section{Pregunta 1}

Para empezar, ¿podría decirme si la situación actual sobre el coronavirus COVID-19, le preocupa a Ud. mucho, bastante, poco o nada?

\begin{tabular}{lc}
\hline Mucho & 58,9 \\
\hline Bastante & 37,0 \\
\hline Regular & 1,2 \\
\hline Poco & 2,6 \\
\hline Nada & 0,4 \\
\hline No tiene información & - \\
\hline Duda & 0,0 \\
\hline N.C. & - \\
\hline (N) & $(3.000)$ \\
\hline
\end{tabular}

\section{Pregunta 2}

¿Cree Ud. que la información que está dando el Gobierno y los/as responsables del tema sobre el COVID-19 es suficiente o le gustaría a Ud. tener más información?

\begin{tabular}{lc}
\hline Cree que es suficiente & 43,4 \\
\hline Le gustaría tener más información & 54,5 \\
\hline No sabe, duda & 1,8 \\
\hline N.C. & 0,3 \\
\hline (N) & $(3.000)$ \\
\hline
\end{tabular}

\section{Pregunta 3}

Respecto a las medidas que se han adoptado en España para combatir el COVID-19, ¿cree Ud. que son muy necesarias, necesarias, poco necesarias o nada necesarias?

\begin{tabular}{lc}
\hline Muy necesarias & 72,6 \\
\hline Necesarias & 24,7 \\
\hline Regular & 0,1 \\
\hline Poco necesarias & 1,6 \\
\hline Nada necesarias & 0,5 \\
\hline No sabe, duda & 0,4 \\
\hline N.C. & 0,1 \\
\hline (N) & $(3.000)$ \\
\hline
\end{tabular}




\section{Pregunta 4}

¿Cree Ud. que deberían tomarse medidas más estrictas para evitar que se salga a la calle, o cree que es suficiente con lo que se está haciendo?

\begin{tabular}{lc}
\hline Cree que deberían tomarse medidas más estrictas & 39,1 \\
\hline Cree que con lo que se está haciendo es suficiente & 58,3 \\
\hline No sabe, duda & 2,4 \\
\hline N.C. & 0,2 \\
\hline (N) & $(3.000)$ \\
\hline
\end{tabular}

\section{Pregunta 5}

Más en concreto, ¿estaría Ud. de acuerdo con que en España no se permitiese salir a la calle y que las medicinas y suministros alimentarios se llevaran a las casas por personal especializado, como se ha hecho en China?

\begin{tabular}{lc}
\hline Está de acuerdo con no permitir la salida de los hogares & 57,7 \\
\hline No está de acuerdo & 38,6 \\
\hline No lo sabe & 3,6 \\
\hline N.C. & 0,1 \\
\hline (N) & $(3.000)$ \\
\hline
\end{tabular}

\section{Pregunta 6}

¿Cree Ud. que en estos momentos habría que prohibir la difusión de bulos e informaciones engañosas y poco fundamentadas por las redes y los medios de comunicación social, remitiendo toda la información sobre la pandemia a fuentes oficiales, o cree que hay que mantener libertad total para la difusión de noticias e informaciones?

\begin{tabular}{lc}
\hline $\begin{array}{l}\text { Cree que habría que restringir y controlar las informaciones, estableciendo sólo una } \\
\text { fuente oficial de información }\end{array}$ & 66,7 \\
\hline Cree que no debe restringirse ni prohibirse ningún tipo de información & 30,8 \\
\hline No lo sabe, duda & 2,0 \\
\hline N.C. & 0,5 \\
\hline (N) & $(3.000)$ \\
\hline
\end{tabular}

\section{Pregunta 7}

La política que está siguiendo el Gobierno actual para luchar contra el COVID-19 en su conjunto, ¿le merece a Ud. mucha confianza, bastante confianza, poca confianza o ninguna confianza?

\begin{tabular}{lc}
\hline Le merece mucha confianza & 10,5 \\
\hline Le merece bastante confianza & 36,0 \\
\hline Regular & 4,8 \\
\hline Le merece poca confianza & 29,8 \\
\hline No le merece ninguna confianza & 18,0 \\
\hline No lo sabe, duda & 0,9 \\
\hline N.C. & 0,1 \\
\hline (N) & $(3.000)$ \\
\hline
\end{tabular}

\section{Pregunta 8}

¿Cree Ud. que en estos momentos es el Gobierno de España el que tiene que tomar las medidas para combatir el COVID-19, o cree que habría que dejar que estas decisiones las tomasen los Gobiernos autonómicos que lo desearan?

\begin{tabular}{lc}
\hline Cree que las medidas las debe tomar el Gobierno de España & 73,3 \\
\hline Cree que las medidas las deberían tomar los Gobiernos autonómicos & 20,7 \\
\hline No sabe, duda & 5,0 \\
\hline N.C. & 0,9 \\
\hline (N) & $(3.000)$ \\
\hline
\end{tabular}




\section{Pregunta 9}

Respecto a las posibilidades de ganar esta batalla contra el COVID-19 en estos momentos, ¿es Ud. muy optimista, bastante optimista, poco optimista o nada optimista?

\begin{tabular}{lc}
\hline Muy optimista & 25,3 \\
\hline Bastante optimista & 51,2 \\
\hline Regular & 4,5 \\
\hline Poco optimista & 16,4 \\
\hline Nada optimista & 2,2 \\
\hline No sabe, duda & 0,4 \\
\hline N.C. & - \\
\hline (N) & $(3.000)$ \\
\hline
\end{tabular}

\section{Pregunta 10}

¿Me podría Ud. decir cómo está llevando personalmente el enclaustramiento en su hogar que se ha acordado? ¿Muy bien, razonablemente bien, bastante mal o muy mal?

\begin{tabular}{lc}
\hline Muy bien & 26,1 \\
\hline Razonablemente bien & 64,9 \\
\hline Regular & 3,1 \\
\hline Bastante mal & 4,1 \\
\hline Muy mal & 1,4 \\
\hline No sabe, duda & 0,3 \\
\hline N.C. & 0,2 \\
\hline (N) & $(3.000)$ \\
\hline
\end{tabular}

\section{Pregunta 11}

Por lo que Ud. está viendo, ¿cree que la mayoría de los/as españoles/as estamos dando un ejemplo de civismo y solidaridad en la forma de afrontar las medidas contra el COVID-19, o piensa que la mayoría está siendo poco cívica e indisciplinada?

\begin{tabular}{lc}
\hline Cree que la mayoría está reaccionando con civismo y solidaridad & 93,5 \\
\hline Cree que la mayoría está siendo poco cívica e indisciplinada & 5,5 \\
\hline No lo sabe, duda & 0,9 \\
\hline N.C. & 0,0 \\
\hline (N) & $(3.000)$ \\
\hline
\end{tabular}

\section{Pregunta 12}

Si en estos momentos tuviéramos en España un Gobierno presidido por Pablo Casado (PP), ¿cree Ud. que la lucha contra el COVID-19 se estaría haciendo mucho mejor, algo mejor, prácticamente igual, algo peor o mucho peor que la que se está haciendo con el Gobierno presidido por Pedro Sánchez (PSOE)?

\begin{tabular}{lc}
\hline Mucho mejor & 7,2 \\
\hline Algo mejor & 11,3 \\
\hline Prácticamente igual & 46,6 \\
\hline Algo peor & 10,0 \\
\hline Mucho peor & 12,9 \\
\hline No sabe, duda & 10,4 \\
\hline N.C. & 1,6 \\
\hline (N) & $(3.000)$ \\
\hline
\end{tabular}




\section{Pregunta 13}

¿Cree Ud. que ante casos de pandemia como la del COVID-19 hay que atenerse a lo que digan y propongan los/as expertos/as en cada momento, o que los Gobiernos tienen que ser capaces de tomar decisiones por su cuenta?

\begin{tabular}{lc}
\hline Hay que atenerse a lo que digan y propongan los/as expertos/as & 88,5 \\
\hline Los Gobiernos tienen que ser capaces de tomar decisiones por su cuenta & 9,2 \\
\hline No sabe, duda & 2,0 \\
\hline N.C. & 0,2 \\
\hline (N) & $(3.000)$ \\
\hline
\end{tabular}

\section{Pregunta 14}

En circunstancias como las actuales, ¿cree Ud. que los partidos y líderes de la oposición tienen que colaborar y apoyar al Gobierno en todo lo posible, dejando sus críticas o discrepancias para otros momentos, o que deben continuar criticando y oponiéndose al actual Gobierno en todo lo que consideren?

\begin{tabular}{lc}
\hline Cree que ahora hay que apoyar al Gobierno y dejar las críticas para otro momento & 87,8 \\
\hline Cree que deben continuar haciendo todas las críticas que consideren oportunas & 10,0 \\
\hline No sabe, duda & 1,7 \\
\hline N.C. & 0,6 \\
\hline (N) & $(3.000)$ \\
\hline
\end{tabular}

\section{Pregunta 15}

¿Cómo cree Ud. que van a ser las consecuencias económicas y laborales que se van a derivar de la crisis del COVID-19, muy graves, algo graves, poco graves o nada graves?

\begin{tabular}{lc}
\hline Muy graves & 80,3 \\
\hline Algo graves & 17,0 \\
\hline Regular & 0,6 \\
\hline Poco graves & 1,5 \\
\hline Nada graves & 0,2 \\
\hline No sabe, duda & 0,4 \\
\hline N.C. & - \\
\hline (N) & $(3.000)$ \\
\hline
\end{tabular}

\section{Pregunta 16}

¿Cree Ud. que cuando termine la crisis del COVID-19 debería hacerse un esfuerzo especial para intentar llegar a grandes acuerdos ante la crisis económica y laboral, o cree que lo mejor es que cada partido plantee sus propias alternativas y puntos de vista?

\begin{tabular}{lc}
\hline Es mejor que se intenten grandes acuerdos & 91,4 \\
\hline Es mejor que cada partido plantee sus propias alternativas y puntos de vista & 6,2 \\
\hline Depende de las circunstancias & 0,5 \\
\hline No sabe, duda & 1,6 \\
\hline N.C. & 0,2 \\
\hline (N) & $(3.000)$ \\
\hline
\end{tabular}

\section{Pregunta 17}

Refiriéndonos a la situación económica general de España al margen del COVID-19, ¿cómo la calificaría Ud.: muy buena, buena, mala o muy mala?

\begin{tabular}{lc}
\hline Muy buena & 1,9 \\
\hline Buena & 52,8 \\
\hline Regular & 11,8 \\
\hline Mala & 26,3 \\
\hline Muy mala & 5,9 \\
\hline N.S. & 1,0 \\
\hline N.C. & 0,3 \\
\hline (N) & $(3.000)$ \\
\hline
\end{tabular}




\section{Pregunta 18}

¿Cree Ud. que dentro de un año la situación económica de España será mejor, igual o peor que ahora?

\begin{tabular}{lr}
\hline Mejor & 26,5 \\
\hline Igual & 16,5 \\
\hline Peor & 53,7 \\
\hline N.S & 3,2 \\
\hline N.C. & - \\
\hline (N) & $(3.000)$ \\
\hline
\end{tabular}

\section{Pregunta 19}

¿Cómo calificaría Ud. su situación económica personal en la actualidad: muy buena, buena, mala o muy mala?

\begin{tabular}{lc}
\hline Muy buena & 4,0 \\
\hline Buena & 65,8 \\
\hline Regular & 10,3 \\
\hline Mala & 15,8 \\
\hline Muy mala & 3,8 \\
\hline N.S. & 0,2 \\
\hline N.C. & 0,1 \\
\hline (N) & $(3.000)$ \\
\hline
\end{tabular}

\section{Pregunta 20}

¿Cree Ud. que dentro de un año su situación económica personal será mejor, igual o peor que ahora?

\begin{tabular}{lr}
\hline Mejor & 15,9 \\
\hline Igual & 56,2 \\
\hline Peor & 24,5 \\
\hline N.S & 3,4 \\
\hline N.C. & 0,1 \\
\hline (N) & $(3.000)$ \\
\hline
\end{tabular}




\section{Pregunta 21}

¿Cuál es, a su juicio, el principal problema que existe actualmente en España? ¿Y el segundo? ¿Y el tercero? (RESPUESTA ESPONTÁNEA). (MULTIRRESPUESTA).

\begin{tabular}{|c|c|c|c|c|}
\hline & $\begin{array}{l}\text { Primer } \\
\text { problema }\end{array}$ & $\begin{array}{l}\text { Segundo } \\
\text { problema }\end{array}$ & $\begin{array}{c}\text { Tercer } \\
\text { problema }\end{array}$ & TOTAL \\
\hline El coronavirus & 42,3 & 5,1 & 1,9 & 49,3 \\
\hline Los problemas de índole económica & 5,5 & 22,6 & 11,0 & 39,1 \\
\hline El paro & 7,7 & 18,6 & 9,7 & 36,0 \\
\hline El mal comportamiento de los/as políticos/as & 9,9 & 6,3 & 4,8 & 20,9 \\
\hline La sanidad & 4,8 & 7,4 & 7,4 & 19,6 \\
\hline Los problemas políticos en general & 7,0 & 4,1 & 4,6 & 15,7 \\
\hline Los problemas de índole social & 1,4 & 3,4 & 5,8 & 10,6 \\
\hline Lo que hacen los partidos políticos & 4,9 & 3,6 & 2,1 & 10,6 \\
\hline El Gobierno, mala gestión del COVID del Gobierno, falta de información & 5,8 & 2,2 & 1,2 & 9,1 \\
\hline Los problemas relacionados con la calidad del empleo & 1,4 & 3,5 & 2,4 & 7,3 \\
\hline Educación & 0,4 & 1,7 & 3,1 & 5,2 \\
\hline La corrupción y el fraude & 1,5 & 2,0 & 1,3 & 4,8 \\
\hline $\begin{array}{l}\text { Los problemas derivados del COVID: incertidumbre, confinamiento, cierre de } \\
\text { negocios, autónomos, PYMES, pocas ayudas }\end{array}$ & 1,2 & 1,4 & 1,9 & 4,4 \\
\hline La independencia de Cataluña & 0,5 & 1,6 & 2,2 & 4,3 \\
\hline Sistema territorial/unidad de España/nacionalismos & 0,8 & 1,1 & 1,6 & 3,4 \\
\hline Los ciudadanos/as, el comportamiento, egoísmo, poco cívicos, bulos & 1,2 & 1,1 & 0,7 & 3,0 \\
\hline Falta de acuerdo entre los/as políticos/as & 1,2 & 1,2 & 0,6 & 3,0 \\
\hline La inmigración & 0,1 & 0,5 & 0,8 & 1,4 \\
\hline Las pensiones & 0,1 & 0,6 & 0,5 & 1,2 \\
\hline La violencia de género & 0,1 & 0,2 & 0,7 & 1,0 \\
\hline Otras respuestas & 0,9 & 2,9 & 4,6 & 8,4 \\
\hline Ninguno & 0,1 & 4,9 & 12,5 & 0,1 \\
\hline N.S. & 1,1 & 2,9 & 9,0 & 1,1 \\
\hline N.C. & 0,1 & 1,3 & 9,7 & 0,1 \\
\hline (N) & $(3.000)$ & $(3.000)$ & $(3.000)$ & $(3.000)$ \\
\hline
\end{tabular}




\section{Pregunta 22}

¿Y cuál es el problema que a Ud., personalmente, le afecta más? ¿Y el segundo? ¿Y el tercero? (RESPUESTA ESPONTÁNEA). (MULTIRRESPUESTA).

\begin{tabular}{|c|c|c|c|c|}
\hline & $\begin{array}{c}\text { Primer } \\
\text { problema }\end{array}$ & $\begin{array}{l}\text { Segundo } \\
\text { problema }\end{array}$ & $\begin{array}{c}\text { Tercer } \\
\text { problema }\end{array}$ & TOTAL \\
\hline El coronavirus & 30,1 & 7,7 & 4,0 & 41,9 \\
\hline Los problemas de índole económica & 12,5 & 15,7 & 6,8 & 34,9 \\
\hline El paro & 10,9 & 10,2 & 4,8 & 25,9 \\
\hline La sanidad & 6,7 & 7,0 & 4,0 & 17,6 \\
\hline Tener que estar enclaustrado en casa & 7,6 & 4,2 & 1,6 & 13,4 \\
\hline Las preocupaciones y situaciones personales & 3,5 & 3,6 & 2,5 & 9,6 \\
\hline La educación & 2,3 & 3,3 & 3,6 & 9,2 \\
\hline $\begin{array}{l}\text { Los problemas derivados del COVID: incertidumbre, futuro, situación autónomos, } \\
\text { PYMES, ERTES }\end{array}$ & 3,1 & 2,8 & 2,5 & 8,4 \\
\hline Los problemas relacionados con la calidad del empleo & 4,3 & 2,7 & 1,4 & 8,3 \\
\hline Los problemas de índole social & 1,8 & 2,9 & 3,1 & 7,7 \\
\hline Los problemas políticos en general & 2,1 & 3,1 & 2,3 & 7,6 \\
\hline El mal comportamiento de los/as políticos/as & 1,5 & 1,5 & 1,9 & 4,9 \\
\hline Los problemas relacionados con la juventud & 1,0 & 1,3 & 0,9 & 3,2 \\
\hline Las pensiones & 1,2 & 1,0 & 0,8 & 3,0 \\
\hline Lo que hacen los partidos políticos & 0,6 & 1,2 & 1,1 & 3,0 \\
\hline El Gobierno, mala gestión del COVID del Gobierno, falta de información & 0,9 & 0,9 & 0,6 & 2,4 \\
\hline La independencia de Cataluña & 0,6 & 0,9 & 0,8 & 2,3 \\
\hline La vivienda & 0,6 & 0,9 & 0,8 & 2,2 \\
\hline La corrupción y el fraude & 0,5 & 0,5 & 0,6 & 1,6 \\
\hline Los/as ciudadanos/as, comportamiento, egoísmo, cada uno/a a lo suyo/poco cívicos & 0,4 & 0,4 & 0,4 & 1,2 \\
\hline Cambio climático, medio ambiente, contaminación, ecosistema & 0,4 & 0,3 & 0,5 & 1,2 \\
\hline Avituallamiento de víveres en el hogar & 0,1 & 0,3 & 0,3 & 0,8 \\
\hline Otras respuestas & 1,8 & 1,7 & 1,8 & 5,4 \\
\hline Ninguno & 4,9 & 16,6 & 19,0 & 4,9 \\
\hline N.S. & 0,6 & 3,5 & 7,1 & 0,6 \\
\hline N.C. & 0,1 & 5,8 & 26,8 & 0,1 \\
\hline (N) & $(3.000)$ & $(3.000)$ & $(3.000)$ & $(3.000)$ \\
\hline
\end{tabular}

\section{Pregunta 23}

A continuación, vamos a mencionarle a Ud. los nombres de algunos/as líderes políticos/as y nos gustaría que, en relación a lo que cada uno/a está diciendo y haciendo sobre el COVID-19 en estos momentos, lo/a calificara de 1 a 10, siendo el 1 'lo peor' y el 10 'lo mejor'.

\begin{tabular}{|c|c|c|c|c|c|c|c|c|c|c|c|c|c|}
\hline & 1 Lo peor & 2 & 3 & 4 & 5 & 6 & 7 & 8 & 9 & 10 Lo mejor & N.S. & N.C. & $(\mathrm{N})$ \\
\hline Pedro Sánchez & 15,9 & 4,7 & 6,1 & 8,1 & 14,1 & 12,1 & 14,9 & 14,0 & 3,3 & 3,6 & 1,8 & 1,4 & $(3.000)$ \\
\hline Pablo Casado & 14,8 & 7,2 & 10,6 & 15,0 & 22,4 & 9,6 & 6,4 & 4,8 & 0,9 & 1,0 & 5,5 & 1,8 & $(3.000)$ \\
\hline Pablo Iglesias & 30,2 & 6,5 & 7,8 & 9,1 & 14,5 & 10,2 & 8,0 & 5,0 & 1,3 & 1,2 & 4,4 & 1,8 & $(3.000)$ \\
\hline Santiago Abascal & 43,6 & 8,1 & 9,2 & 7,9 & 9,7 & 3,8 & 3,3 & 2,3 & 0,8 & 1,4 & 8,0 & 1,8 & $(3.000)$ \\
\hline Inés Arrimadas & 17,1 & 8,8 & 10,1 & 12,6 & 19,2 & 8,5 & 5,0 & 3,2 & 0,8 & 0,6 & 12,5 & 1,7 & $(3.000)$ \\
\hline \multirow[t]{2}{*}{ Alberto Garzón } & 19,8 & 6,7 & 8,2 & 9,7 & 14,6 & 7,5 & 5,4 & 2,9 & 1,0 & 0,8 & 21,7 & 1,8 & $(3.000)$ \\
\hline & Media & \multicolumn{2}{|c|}{$\begin{array}{c}\text { Desviación } \\
\text { típica }\end{array}$} & \multicolumn{2}{|c|}{ (N) } & & & & & & & & \\
\hline Pedro Sánchez & 5,2 & & 2,6 & \multicolumn{2}{|c|}{$(2.904)$} & & & & & & & & \\
\hline Pablo Casado & 4,2 & & 2,1 & \multicolumn{2}{|c|}{$(2.781)$} & & & & & & & & \\
\hline Pablo Iglesias & 3,8 & & 2,5 & \multicolumn{2}{|c|}{$(2.814)$} & & & & & & & & \\
\hline Santiago Abascal & 2,8 & & 2,3 & \multicolumn{2}{|c|}{$(2.705)$} & & & & & & & & \\
\hline Inés Arrimadas & 3,9 & & 2,1 & \multicolumn{2}{|c|}{$(2.576)$} & & & & & & & & \\
\hline Alberto Garzón & 3,8 & & 2,3 & \multicolumn{2}{|c|}{$(2.295)$} & & & & & & & & \\
\hline
\end{tabular}




\section{Pregunta 24}

El presidente del Gobierno, Pedro Sánchez, ¿le inspira a Ud., personalmente, mucha confianza, bastante confianza, poca o ninguna confianza?

\begin{tabular}{lr}
\hline Mucha confianza & 7,4 \\
\hline Bastante confianza & 31,6 \\
\hline Poca confianza & 31,9 \\
\hline Ninguna confianza & 28,1 \\
\hline N.S. & 0,4 \\
\hline N.C. & 0,6 \\
\hline (N) & $(3.000)$ \\
\hline
\end{tabular}

\section{Pregunta 25}

¿Y el líder del principal partido de la oposición, Pablo Casado, le inspira, personalmente, mucha confianza, bastante confianza, poca o ninguna confianza?

\begin{tabular}{lr}
\hline Mucha confianza & 2,4 \\
\hline Bastante confianza & 18,2 \\
\hline Poca confianza & 42,9 \\
\hline Ninguna confianza & 34,1 \\
\hline N.S. & 1,4 \\
\hline N.C. & 0,9 \\
\hline (N) & $(3.000)$ \\
\hline
\end{tabular}

\section{Pregunta 26R}

De los/as principales líderes políticos/as, ¿quién preferiría que fuese el/la presidente/a del Gobierno en estos momentos? (RESPUESTA ESPONTÁNEA). (RECODIFICADA).

\begin{tabular}{lc}
\hline Pedro Sánchez & 35,5 \\
\hline Pablo Casado & 12,5 \\
\hline Santiago Abascal & 6,1 \\
\hline Pablo Iglesias & 4,5 \\
\hline Alberto Garzón & 1,3 \\
\hline Inés Arrimadas & 6,0 \\
\hline Miguel Ángel Revilla & 0,8 \\
\hline Alberto Núñez Feijóo & 1,1 \\
\hline (NO LEER) Otro/a & 5,4 \\
\hline (NO LEER) Ninguno/a de ellos/as & 19,7 \\
\hline N.S. & 6,0 \\
\hline N.C. & 1,1 \\
\hline (N) & $(3.000)$ \\
\hline
\end{tabular}




\section{Pregunta 27R}

Suponiendo que mañana se celebrasen nuevamente elecciones generales, es decir, al Parlamento español, ¿a qué partido votaría Ud.? (RESPUESTA ESPONTÁNEA). (RECODIFICADA).

\begin{tabular}{lc}
\hline PSOE & 25,0 \\
\hline PP & 14,8 \\
\hline VOX & 7,1 \\
\hline Unidas Podemos & 9,3 \\
\hline Ciudadanos & 5,8 \\
\hline Más País & 0,5 \\
\hline ERC & 2,4 \\
\hline JxCat & 1,0 \\
\hline CUP & 0,4 \\
\hline EAJ-PNV & 0,9 \\
\hline EH Bildu & 0,3 \\
\hline CCa-NC & 0,2 \\
\hline NAt & 0,2 \\
\hline Compromís & 0,7 \\
\hline BNG & 0,4 \\
\hline PRC & 0,1 \\
\hline Teruel Existe & 0,0 \\
\hline PACMA & 0,8 \\
\hline Otro partido & 0,8 \\
\hline Voto blanco & 4,7 \\
\hline Voto nulo & 1,5 \\
\hline No votaría & 9,1 \\
\hline No sabe todavía & 10,9 \\
\hline N.C. & 3,0 \\
\hline (N) & $(3.000)$ \\
\hline
\end{tabular}


SÓLO A QUIENES VOTARÍAN A ALGÚN PARTIDO EN UNAS SUPUESTAS ELECCIONES GENERALES

(A todos excepto 'Voto nulo', 'En blanco', 'No votaría', 'No sabe todavía' o 'N.C.' en P27)

$(\mathrm{N}=2.123)$

\section{Pregunta 27aR}

En el caso de que por cualquier razón finalmente no votase por el partido que me ha dicho, ¿a qué otro partido votaría Ud.? (RESPUESTA ESPONTÁNEA). (RECODIFICADA).

\begin{tabular}{lc}
\hline PSOE & 12,0 \\
\hline PP & 13,0 \\
\hline VOX & 6,1 \\
\hline Unidas Podemos & 11,7 \\
\hline En Comú Podem & 3,3 \\
\hline En Común-Unidas Podemos & 1,4 \\
\hline Ciudadanos & 14,9 \\
\hline Más País & 0,6 \\
\hline ERC & 2,3 \\
\hline JxCat & 1,3 \\
\hline CUP & 0,7 \\
\hline EAJ-PNV & 0,7 \\
\hline EH Bildu & 0,4 \\
\hline CCa-NC & 0,5 \\
\hline NAt & 0,4 \\
\hline Compromís & 0,8 \\
\hline BNG & 0,7 \\
\hline PRC & 0,0 \\
\hline PACMA & 1,6 \\
\hline Otro partido & 2,3 \\
\hline Voto blanco & 6,1 \\
\hline No votaría & 12,0 \\
\hline N.S. & 7,3 \\
\hline N.C. & 0,2 \\
\hline (N) & $(2.123)$ \\
\hline & \\
\hline
\end{tabular}

\section{SÓLO A QUIENES NO VOTARÍAN A NINGÚN PARTIDO EN UNAS SUPUESTAS ELECCIONES GENERALES}

(Contestan 'Voto nulo', 'En blanco', 'No votaría', 'No sabe todavía' o 'N.C.' en P27)

$(\mathrm{N}=877)$

\section{Pregunta 28R}

Sin ningún compromiso por su parte, ¿me podría decir por qué partido siente Ud. más simpatía? (RESPUESTA ESPONTÁNEA). (RECODIFICADA).

\begin{tabular}{lc}
\hline PSOE & 16,8 \\
\hline PP & 7,8 \\
\hline Podemos & 3,4 \\
\hline Ciudadanos & 6,8 \\
\hline ERC & 1,6 \\
\hline Otro partido & 7,9 \\
\hline Ninguno & 43,7 \\
\hline N.S. & 4,3 \\
\hline N.C. & 7,8 \\
\hline (N) & $(877)$ \\
\hline
\end{tabular}




\section{Pregunta 28a}

\section{VOTO+SIMPATÍA (ELECCIONES GENERALES).}

\begin{tabular}{lc}
\hline PSOE & 28,8 \\
\hline PP & 16,7 \\
\hline VOX & 7,5 \\
\hline Unidas Podemos & 10,5 \\
\hline Ciudadanos & 7,2 \\
\hline Más País & 0,5 \\
\hline ERC & 2,8 \\
\hline JxCat & 1,0 \\
\hline CUP & 0,5 \\
\hline EAJ-PNV & 1,2 \\
\hline EH Bildu & 0,3 \\
\hline CCa-NC & 0,3 \\
\hline NAt & 0,2 \\
\hline Compromís & 0,7 \\
\hline BNG & 0,5 \\
\hline PRC & 0,2 \\
\hline Teruel Existe & 0,1 \\
\hline PACMA & 1,1 \\
\hline Otro partido & 1,1 \\
\hline En blanco & 4,7 \\
\hline Voto nulo & 1,5 \\
\hline No votaría & 9,5 \\
\hline No sabe todavía & 1,0 \\
\hline N.C. & 2,2 \\
\hline (N) & $(3.000)$ \\
\hline
\end{tabular}

\section{Pregunta 29}

Cuando se habla de política se utilizan normalmente las expresiones izquierda y derecha. Situándonos en una escala de 10 casillas, como un termómetro, que van del 1 al 10 en la que 1 significa 'lo más a la izquierda' y 10 'lo más a la derecha', ¿en qué casilla se colocaría Ud.?

\begin{tabular}{lr}
\hline 1 Izquierda & 8,9 \\
\hline 2 & 5,3 \\
\hline 3 & 12,3 \\
\hline 4 & 12,4 \\
\hline 5 & 30,9 \\
\hline 6 & 10,0 \\
\hline 7 & 7,0 \\
\hline 8 & 5,3 \\
\hline 9 & 1,1 \\
\hline 10 Derecha & 3,2 \\
\hline N.S. & 2,0 \\
\hline N.C. & 1,8 \\
\hline (N) & $(3.000)$ \\
\hline Media & 4,7 \\
\hline Desviación típica & 2,1 \\
\hline (N) & $(2.886)$ \\
\hline
\end{tabular}




\section{Pregunta 30}

Y siguiendo este mismo criterio, a los/as líderes de los principales partidos políticos, ¿dónde los/as ubicaría?

\begin{tabular}{|c|c|c|c|c|c|c|c|c|c|c|c|c|c|}
\hline & 1 Izquierda & 2 & 3 & 4 & 5 & 6 & 7 & 8 & 9 & 10 Derecha & N.S. & N.C. & (N) \\
\hline Pedro Sánchez & 14,0 & 8,6 & 20,6 & 19,6 & 19,5 & 7,3 & 3,0 & 1,1 & 0,2 & 0,9 & 3,5 & 1,6 & $(3.000)$ \\
\hline Pablo Casado & 0,9 & 0,7 & 0,5 & 1,7 & 8,2 & 11,2 & 21,4 & 24,3 & 11,0 & 14,1 & 4,3 & 1,7 & $(3.000)$ \\
\hline Santiago Abascal & 1,4 & 0,5 & 0,6 & 1,2 & 3,6 & 2,6 & 3,5 & 8,1 & 11,5 & 57,7 & 7,4 & 1,9 & $(3.000)$ \\
\hline Pablo Iglesias & 42,7 & 18,8 & 13,2 & 7,1 & 6,9 & 2,3 & 1,2 & 0,9 & 0,2 & 0,4 & 4,4 & 1,9 & $(3.000)$ \\
\hline Alberto Garzón & 26,0 & 19,2 & 13,3 & 7,2 & 9,0 & 2,6 & 1,4 & 0,7 & 0,3 & 0,4 & 18,2 & 1,8 & $(3.000)$ \\
\hline Inés Arrimadas & 2,0 & 1,5 & 2,8 & 4,0 & 19,3 & 20,7 & 16,5 & 9,5 & 5,6 & 5,4 & 11,1 & 1,5 & $(3.000)$ \\
\hline
\end{tabular}

\begin{tabular}{lccc}
\hline & Media & $\begin{array}{c}\text { Desviación } \\
\text { típica }\end{array}$ & (N) \\
\hline Pedro Sánchez & 3,7 & 1,8 & $(2.846)$ \\
\hline Pablo Casado & 7,5 & 1,8 & $(2.820)$ \\
\hline Santiago Abascal & 9,0 & 1,9 & $(2.720)$ \\
\hline Pablo Iglesias & 2,3 & 1,7 & $(2.811)$ \\
\hline Alberto Garzón & 2,7 & 1,8 & $(2.402)$ \\
\hline Inés Arrimadas & 6,3 & 1,9 & $(2.624)$ \\
\hline
\end{tabular}

\section{Pregunta 31}

Por otro lado, le agradecería que me indicara si conoce a cada uno/a de los/as siguientes líderes políticos/as y qué valoración le merece. Puntúelos/as de 1 a 10, sabiendo que el 1 significa que lo/a valora 'muy mal' y el 10 que lo/a valora 'muy bien'.

\begin{tabular}{lrrrrrrrrrrrrrr}
\hline & 1 Muy mal & 2 & 3 & 4 & 5 & 6 & 7 & 8 & 9 & 10 Muy bien & No conoce & N.S. & N.C. & (N) \\
\hline Pedro Sánchez & 17,5 & 6,0 & 7,4 & 8,7 & 13,5 & 11,8 & 14,4 & 11,6 & 3,1 & 4,1 & 0,4 & 0,4 & 1,1 & $(3.000)$ \\
\hline Pablo Casado & 14,9 & 9,7 & 13,4 & 15,0 & 17,8 & 10,2 & 7,3 & 5,1 & 1,7 & 1,6 & 1,6 & 0,7 & 1,0 & $(3.000)$ \\
\hline Santiago Abascal & 45,9 & 7,8 & 8,5 & 7,2 & 8,4 & 5,1 & 4,1 & 3,7 & 1,4 & 1,9 & 3,9 & 1,0 & 1,2 & $(3.000)$ \\
\hline Pablo Iglesias & 32,5 & 8,0 & 9,3 & 9,5 & 12,0 & 8,2 & 9,1 & 5,1 & 1,9 & 1,5 & 1,0 & 0,6 & 1,1 & $(3.000)$ \\
\hline Alberto Garzón & 21,4 & 7,7 & 9,8 & 8,9 & 12,2 & 8,1 & 7,3 & 4,8 & 1,7 & 1,5 & 13,7 & 1,7 & 1,1 & $(3.000)$ \\
\hline Inés Arrimadas & 12,6 & 8,3 & 11,6 & 15,0 & 18,1 & 11,2 & 7,3 & 4,1 & 1,5 & 1,2 & 6,9 & 1,1 & 1,1 & $(3.000)$ \\
\hline
\end{tabular}

\begin{tabular}{lccc}
\hline & Media & $\begin{array}{c}\text { Desviación } \\
\text { típica }\end{array}$ & (N) \\
\hline Pedro Sánchez & 5,0 & 2,7 & $(2.942)$ \\
\hline Pablo Casado & 4,2 & 2,2 & $(2.899)$ \\
\hline Santiago Abascal & 3,0 & 2,5 & $(2.818)$ \\
\hline Pablo Iglesias & 3,7 & 2,6 & $(2.918)$ \\
\hline Alberto Garzón & 4,0 & 2,5 & $(2.503)$ \\
\hline Inés Arrimadas & 4,3 & 2,2 & $(2.727)$ \\
\hline
\end{tabular}

\section{Pregunta 32}

¿Me podría decir si en las elecciones generales del pasado 10 de noviembre de 2019...?

\begin{tabular}{lc}
\hline Fue a votar y votó/Votó por correo & 87,6 \\
\hline No tenía edad para votar & 0,3 \\
\hline Fue a votar pero no pudo hacerlo & 0,2 \\
\hline No fue a votar porque no pudo & 4,5 \\
\hline Prefirió no votar & 6,8 \\
\hline No tenía derecho a voto & 0,1 \\
\hline No recuerda & 0,1 \\
\hline N.C. & 0,3 \\
\hline (N) & $(3.000)$ \\
\hline
\end{tabular}


SÓLO A QUIENES VOTARON EN LAS ELECCIONES GENERALES DE NOVIEMBRE DE 2019

(1 en P32)

$(\mathrm{N}=2.629)$

\section{Pregunta 32aR}

¿Y podría decirme a qué partido o coalición votó? (RESPUESTA ESPONTÁNEA). (RECODIFICADA).

\begin{tabular}{lc}
\hline PSOE & 28,9 \\
\hline PP & 14,6 \\
\hline VOX & 7,6 \\
\hline Unidas Podemos & 8,3 \\
\hline En Comú Podem & 2,4 \\
\hline En Común-Unidas Podemos & 1,0 \\
\hline ERC & 2,5 \\
\hline Ciudadanos & 8,0 \\
\hline JxCat & 1,5 \\
\hline EAJ-PNV & 1,3 \\
\hline EH Bildu & 0,6 \\
\hline Más País & 0,8 \\
\hline CUP & 0,8 \\
\hline CCa-NC & 0,5 \\
\hline NAt & 0,3 \\
\hline Més Compromís & 0,9 \\
\hline BNG & 0,5 \\
\hline PRC & 0,1 \\
\hline Teruel Existe & 0,1 \\
\hline PACMA & 0,8 \\
\hline Otros partidos & 0,9 \\
\hline En blanco & 3,3 \\
\hline Voto nulo & 1,6 \\
\hline No recuerda & 1,7 \\
\hline N.C. & 10,8 \\
\hline (N) & $(2.629)$ \\
\hline
\end{tabular}




\section{Pregunta 32aa}

RECUERDO DE VOTO EN ELECCIONES GENERALES DE NOVIEMBRE DE 2019.

\begin{tabular}{lc}
\hline PSOE & 25,3 \\
\hline PP & 12,8 \\
\hline VOX & 6,7 \\
\hline Unidas Podemos & 7,3 \\
\hline En Comú Podem & 2,1 \\
\hline En Común-Unidas Podemos & 0,9 \\
\hline ERC & 2,2 \\
\hline Ciudadanos & 7,0 \\
\hline JxCat & 1,3 \\
\hline EAJ-PNV & 1,1 \\
\hline EH Bildu & 0,5 \\
\hline Más País & 0,7 \\
\hline CUP & 0,7 \\
\hline CCa-NC & 0,4 \\
\hline NAt & 0,3 \\
\hline Més Compromís & 0,8 \\
\hline BNG & 0,5 \\
\hline PRC & 0,1 \\
\hline Teruel Existe & 0,1 \\
\hline PACMA & 0,7 \\
\hline Otros partidos & 0,8 \\
\hline En blanco & 2,9 \\
\hline Nulo & 1,4 \\
\hline No tenía edad & 0,3 \\
\hline No votó & 11,5 \\
\hline No tenía derecho a voto & 0,1 \\
\hline No recuerda & 1,6 \\
\hline N.C. & 9,8 \\
\hline N) & \\
\hline & \\
\hline
\end{tabular}

\section{Pregunta 33R}

En todo caso, ¿cuál de los siguientes partidos considera más cercano a sus propias ideas? (RESPUESTA ESPONTÁNEA). (RECODIFICADA).

\begin{tabular}{lc}
\hline PSOE & 28,8 \\
\hline PP & 15,2 \\
\hline VOX & 5,8 \\
\hline Podemos & 5,8 \\
\hline IU & 1,3 \\
\hline Ciudadanos & 10,9 \\
\hline Más País & 0,6 \\
\hline ERC & 2,4 \\
\hline JxCat & 1,5 \\
\hline CUP & 0,5 \\
\hline EAJ-PNV & 1,1 \\
\hline EH Bildu & 0,6 \\
\hline Compromís & 0,9 \\
\hline PACMA & 0,5 \\
\hline Unidas Podemos & 4,8 \\
\hline Otro partido & 2,0 \\
\hline Ninguno & 11,7 \\
\hline N.S. & 2,5 \\
\hline N.C. & 3,0 \\
\hline (N) & $(3.000)$ \\
\hline
\end{tabular}




\section{Pregunta 34}

¿Ha ido Ud. a la escuela o cursado algún tipo de estudios?

\begin{tabular}{lr}
\hline No, es analfabeto/a & 0,1 \\
\hline No, pero sabe leer y escribir & 1,0 \\
\hline Sí, ha ido a la escuela & 98,9 \\
\hline N.C. & 0,1 \\
\hline (N) & $(3.000)$ \\
\hline
\end{tabular}

\section{SÓLO A QUIENES HAN IDO A LA ESCUELA \\ (3 en P34) \\ $(\mathrm{N}=2.966)$}

\section{Pregunta 34a}

¿Cuáles son los estudios de más alto nivel oficial que Ud. ha cursado (con independencia de que los haya terminado 0 no)?

\begin{tabular}{|c|c|}
\hline Menos de 5 años de escolarización & 0,5 \\
\hline $\begin{array}{l}\text { Educación primaria (Educación primaria de LOGSE, } 5^{\circ} \text { Curso de EGB, Enseñanza } \\
\text { primaria antigua) }\end{array}$ & 8,6 \\
\hline $\begin{array}{l}\text { Cualificación profesional grado inicial (FP grado inicial). PCPI (Programas de } \\
\text { Cualificación Profesional Inicial, que no precisan de titulación académica de la } \\
\text { primera etapa de secundaria para su realización). Programas de garantía social }\end{array}$ & 1,1 \\
\hline $\begin{array}{l}\text { Educación secundaria (ESO, EGB. Graduado Escolar. Certificado de Escolaridad, } \\
\text { Bachillerato Elemental) }\end{array}$ & 13,0 \\
\hline $\begin{array}{l}\text { FP de grado medio (Ciclo/módulo formativo de FP (grado medio), de Artes Plásticas } \\
\text { y Diseño, Música y danza, Enseñanzas deportivas, FP I, Bachiller laboral elemental. } \\
\text { Oficialía Industrial; Bachillerato Comercial) }\end{array}$ & 8,0 \\
\hline $\begin{array}{l}\text { Bachillerato (Bachillerato LOGSE, BUP, Bachillerato superior }\left(6^{\circ}\right) \text {, Bachillerato } \\
\text { universitario }\left(7^{\circ}\right) \text {, incluidos COU y PREU) }\end{array}$ & 13,8 \\
\hline $\begin{array}{l}\text { FP de grado superior (Ciclo/módulo formativo de FP (grado superior) de Artes } \\
\text { Plásticas, Diseño, Música y danza, Deporte, FP II, Bach. Laboral Sup., Maestría } \\
\text { industrial, Perito Mercantil; Secretariado de } 2^{\circ} \text { grado; Grado Medio conservatorio) }\end{array}$ & 13,3 \\
\hline Arquitectura-ingeniería técnica (Arquitectura/ingeniería técnica, Aparejador; Peritos) & 0,8 \\
\hline $\begin{array}{l}\text { Diplomatura (ATENCIÓN: solo Diplomaturas oficiales, no codificar aquí los tres } \\
\text { primeros años de una licenciatura o grado con mayor duración) }\end{array}$ & 8,5 \\
\hline Grado (Estudios de grado, Enseñanzas Artísticas equivalentes (desde 2006)) & 5,3 \\
\hline $\begin{array}{l}\text { Licenciatura (Titulaciones con equivalencia oficial: } 2^{0} \text { ciclo INEF; Danza y arte } \\
\text { dramático (desde 1992); Grado superior de música) }\end{array}$ & 16,9 \\
\hline Arquitectura/ingeniería & 1,8 \\
\hline Máster oficial universitario (Especialidades médicas o equivalente) & 5,6 \\
\hline Doctorado & 1,6 \\
\hline Títulos propios de posgrado (máster no oficial, etc.) & 0,5 \\
\hline Otros estudios & 0,6 \\
\hline N.S./No recuerda & 0,1 \\
\hline N.C. & 0,1 \\
\hline (N) & $(2.966)$ \\
\hline
\end{tabular}

\section{Pregunta 34aa}

\section{ESTUDIOS.}

\begin{tabular}{lc}
\hline Sin estudios & 1,5 \\
\hline Primaria & 8,5 \\
\hline Secundaria $1^{\mathrm{a}}$ etapa & 12,8 \\
\hline Secundaria $2^{\mathrm{a}}$ etapa & 13,7 \\
\hline F.P. & 22,1 \\
\hline Superiores & 40,6 \\
\hline Otros & 0,6 \\
\hline N.C. & 0,3 \\
\hline (N) & $(3.000)$ \\
\hline
\end{tabular}




\section{Pregunta 35}

¿Cómo se define Ud. en materia religiosa: católico/a practicante, católico/a no practicante, creyente de otra religión, agnóstico/a, indiferente o no creyente, o ateo/a?

\begin{tabular}{lc}
\hline Católico/a practicante & 19,5 \\
\hline Católico/a no practicante & 41,7 \\
\hline Creyente de otra religión & 1,8 \\
\hline Agnóstico/a & 11,1 \\
\hline Indiferente, no creyente & 11,5 \\
\hline Ateo/a & 13,5 \\
\hline N.C. & 0,8 \\
\hline (N) & $(3.000)$ \\
\hline
\end{tabular}

\section{Pregunta 36}

¿Cuál es su estado civil?

\begin{tabular}{lc}
\hline Casado/a & 56,0 \\
\hline Soltero/a & 30,6 \\
\hline Viudo/a & 6,2 \\
\hline Separado/a & 1,6 \\
\hline Divorciado/a & 5,1 \\
\hline N.C. & 0,4 \\
\hline (N) & $(3.000)$ \\
\hline
\end{tabular}

\section{Pregunta 37}

¿En qué situación laboral se encuentra Ud. actualmente?

\begin{tabular}{lc}
\hline Trabaja & 51,1 \\
\hline Jubilado/a o pensionista (anteriormente ha trabajado) & 24,5 \\
\hline Pensionista (anteriormente no ha trabajado) & 1,6 \\
\hline En paro y ha trabajado antes & 12,1 \\
\hline En paro y busca su primer empleo & 0,2 \\
\hline Estudiante & 5,3 \\
\hline Trabajo doméstico no remunerado & 3,4 \\
\hline Otra situación & 1,4 \\
\hline N.C. & 0,4 \\
\hline (N) & $(3.000)$ \\
\hline
\end{tabular}

SÓLO A QUIENES TRABAJAN ACTUALMENTE

(1 en P37)

$(\mathrm{N}=1.534)$

\section{Pregunta 37a}

¿Me puede decir cuál es su ocupación actual?

\begin{tabular}{lc}
\hline Directores/as y gerentes & 8,8 \\
\hline Profesionales científicos e intelectuales & 15,1 \\
\hline Técnicos/as y profesionales de nivel medio & 24,0 \\
\hline Personal de apoyo administrativo & 11,8 \\
\hline Trabajadores/as de los servicios y vendedores/as de comercios y mercados & 14,3 \\
\hline $\begin{array}{l}\text { Agricultores/as y trabajadores/as cualificados/as agropecuarios, forestales y } \\
\text { pesqueros }\end{array}$ & 3,1 \\
\hline $\begin{array}{l}\text { Oficiales/as, operarios/as, artesanos/as y trabajadores/as de artes mecánicas y de } \\
\text { otros oficios }\end{array}$ & 6,6 \\
\hline Operadores/as de instalaciones y máquinas y ensambladores/as & 3,4 \\
\hline Ocupaciones elementales & 8,0 \\
\hline Ocupaciones militares y cuerpos policiales & 1,2 \\
\hline Otra/o & 3,4 \\
\hline N.C. & 0,4 \\
\hline (N) & $1.534)$ \\
\hline
\end{tabular}




\section{Pregunta 38}

¿A qué clase social diría Ud. que pertenece? (RESPUESTA ESPONTÁNEA).

\begin{tabular}{lc}
\hline Clase alta & 0,3 \\
\hline Clase media-alta & 11,0 \\
\hline Clase media-media & 45,3 \\
\hline Clase media-baja & 22,7 \\
\hline Clase trabajadora/obrera & 10,0 \\
\hline Clase baja & 5,7 \\
\hline Clase pobre & 0,7 \\
\hline Proletariado & 0,4 \\
\hline A la gente común & 0,7 \\
\hline Otra & 0,8 \\
\hline No cree en las clases & 0,3 \\
\hline No sabe/duda & 1,3 \\
\hline N.C. & 0,8 \\
\hline (N) & $(3.000)$ \\
\hline
\end{tabular}

\section{Pregunta 38a}

\section{IDENTIFICACIÓN SUBJETIVA DE CLASE.}

\begin{tabular}{lc}
\hline Clase alta y media alta & 11,3 \\
\hline Clase media-media & 45,3 \\
\hline Clase media-baja & 22,7 \\
\hline Clase trabajadora/obrera/proletariado & 10,4 \\
\hline Clase baja/pobre & 6,3 \\
\hline Otras & 1,7 \\
\hline N.S. & 1,3 \\
\hline N.C. & 0,8 \\
\hline N) & $(3.000)$ \\
\hline
\end{tabular}


CIS

Centro de Investigaciones Sociológicas

\section{ESTIMACIÓN DE VOTO}

Estudio $n^{\circ} 3279$

Barómetro especial de abril 2020 


\section{NOTA METODOLÓGICA}

Como informamos en septiembre de 2018, el desajuste existente entre los modelos tradicionales de estimación de voto empleados por el CIS hasta julio de 2018 aconsejaba que el Departamento de Investigación iniciara un programa de optimización de los modelos de estimación que recogiera de forma válida y fiable la nueva diversidad política existente en España. Los hechos demostraban que, aunque los modelos tradicionales habían sido apropiados para sistemas de competición básicamente bipartidistas, ya no reflejaban adecuadamente la realidad empírica multipartidista que había emergido tras las elecciones de 2015.

De acuerdo con los criterios habituales en la actividad científica, el diseño y testado de los nuevos modelos de estimación de voto se ha efectuado de forma trasparente y pública, permitiendo a los especialistas en investigación social que así lo han deseado aportar sus contribuciones en un debate alejado de intereses particulares, o focos mediáticos. El CIS agradece todas las contribuciones y consideraciones metodológicas de los investigadores que han propuesto, con buena voluntad, líneas de mejora y optimización en los procesos de medición.

Durante el período transcurrido desde entonces, con el fin de no confundir a la opinión pública nos hemos limitado a ofrecer datos brutos directos de intención de voto declarados. En este período de tiempo hemos constatado que el modelo de estimación que ha mostrado una mayor robustez, aún en condiciones de alta volatilidad y variabilidad en el número de partidos concurrentes, es el que se corresponde con la lógica del diseño V108. Diseño que se ha documentado de forma detallada en los diversos momentos de su testado empírico en las últimas encuestas pre y postelectorales del CIS.

Es preciso aclarar que desde septiembre de 2018 el CIS no ha modificado varias veces los modelos de estimación de voto, como algunos han sostenido erróneamente, sino que solo ha habido dos variaciones sustantivas en la presentación de los datos. Una de ellas ha consistido en la presentación bruta directa de los datos, tal como los expresaban los encuestados directamente, aportando con rapidez y transparencia toda la información sociológica obtenida, para que cualquiera pudiera efectuar estimaciones propias, si así lo deseaba. Y la otra ha consistido en la presentación de los datos ponderados de acuerdo con los nuevos enfoques que han terminado concretándose en el modelo V108 (en las encuestas pre y postelectorales). Modelo que desde enero 2020 hemos incorporado a la práctica del CIS de manera permanente, con los ajustes y adecuaciones que han venido aconsejadas por los procesos de verificación, contraste y testado de este modelo y que son propios de la investigación científica.

El modelo V108 se incorpora a los ya existentes en el CIS y será utilizado para establecer la serie de monitorización de tendencias en el comportamiento electoral que se ha iniciado desde el barómetro de enero 2020. Lógicamente, se trata de un modelo sometido a eventuales verificaciones y contrastes empíricos ulteriores, propios de una realidad sociológica cambiante, cuya intensidad y dinamismo exige las correspondientes adecuaciones empíricas y metodológicas que procedan en función de los nuevos cambios que puedan producirse en las realidades sociopolíticas que se estudian.

Este modelo en ningún caso aspira a representar un "modelo anticipado de estimación de voto". Evidentemente la aplicación de modelos diferentes puede dar lugar a resultados también diferentes. Dado el carácter público de los datos del CIS, los investigadores y demás profesionales pueden producir y difundir bajo su responsabilidad los modelos y estimaciones que consideren más convenientes. 


\begin{tabular}{|c|c|c|}
\hline \multicolumn{3}{|c|}{ Barómetro CIS especial de abril 2020} \\
\hline & $\begin{array}{c}\text { Voto directo } \\
\text { en la } \\
\text { encuesta } \\
\text { (en \% sobre } \\
\text { censo) }\end{array}$ & $\begin{array}{c}\text { Estimación de } \\
\text { voto CIS } \\
\text { (en \% sobre el } \\
\text { voto válido) }\end{array}$ \\
\hline PSOE & 25,0 & 31,2 \\
\hline$P P$ & 14,8 & 21,1 \\
\hline VOx & 7,1 & 13,0 \\
\hline $\begin{array}{l}\text { Unidas Podemos } \\
\text { (En Comú Podem, } \\
\text { En Común-Unidas Podemos) }\end{array}$ & 9,3 & 12,0 \\
\hline Ciudadanos & 5,8 & 7,6 \\
\hline$E R C$ & 2,4 & 3,3 \\
\hline JxCat & 1,0 & 1,4 \\
\hline$E A J-P N V$ & 0,9 & 1,2 \\
\hline EH Bildu & 0,3 & 0,5 \\
\hline Más País / Compromís & 1,2 & 1,5 \\
\hline CUP & 0,4 & 0,6 \\
\hline CCa-NC & 0,2 & 0,4 \\
\hline NA+ & 0,2 & 0,3 \\
\hline$B N G$ & 0,4 & 0,5 \\
\hline PRC & 0,1 & 0,2 \\
\hline Teruel Existe & 0,0 & 0,1 \\
\hline PACMA & 0,8 & 1,1 \\
\hline Otros partidos & 0,8 & 1,1 \\
\hline En blanco & 4,7 & 2,9 \\
\hline Voto nulo & 1,5 & \\
\hline $\begin{array}{l}\text { Abstención } \\
\text { ("No votaría") }\end{array}$ & 9,1 & \\
\hline No sabe & 10,9 & \\
\hline No contesta & 3,0 & \\
\hline
\end{tabular}

- Como consecuencia del efecto de redondeo del segundo decimal el sumatorio puede no ser exactamente 100 . 